\title{
Newborn with rhizomelia and difficulty breathing
}

\author{
Evelina Maines ${ }^{1} \cdot$ Laura Baggio $^{2} \cdot$ Giorgia Gugelmo $^{1} \cdot$ Grazia Morandi $^{2}$. \\ Andrea Bordugo ${ }^{1}$
}

Published online: 1 December 2016

(C) ISS 2016

Chest radiograph highlighted bilateral shortening of the humerus, periarticular and epiphyseal calcifications, large and irregular metaphyses and slightly narrow chest, suggesting the diagnosis of rhizomelic chondrodysplasia punctata (RCDP), a rare autosomal recessive peroxisomal disease with an estimated incidence of 1:100,000 births [1].

The radiograph of the entire skeleton confirmed symmetrical proximal shortness (rhizomelia) with broad metaphyses of long bones, extensive punctate calcification, absence of pubic ossification, and deformities of both feet, including calcaneal stippling.

On the physical examination, the patient presented proximal shortening of both upper and lower limbs, mild dysmorphic features (prominent forehead and depressed nasal bridge), bilat-

The case presentation can be found at doi: 10.1007/s00256-016-2536-y

Evelina Maines

evelina.maines@gmail.com

Laura Baggio

baggio.laura7@gmail.com

Giorgia Gugelmo

gugelmogiorgia@gmail.com

Grazia Morandi

grazia.morandi@gmail.com

Andrea Bordugo

andrea.bordugo@ospedaleuniverona.it

1 Department of Pediatrics, Regional Centre for Newborn Screening, Diagnosis and Treatment of Inherited Metabolic Diseases and Congenital Endocrine Diseases, Azienda Ospedaliera Universitaria Integrata of Verona, Piazzale Ludovico Antonio Scuro, 10, 37134 Verona, Italy

2 Pediatric Clinic, Department of Life and Reproduction Sciences, University of Verona, Verona, Italy eral cataracts, mild flexion contractures of inferior limbs, and bilateral talipes calcaneovalgus. Ichthyosis was not present.

The patient was the third sibling of two consanguineous Pakistani parents. The first daughter died at 15 days of life because of respiratory failure associated with unspecified skeletal abnormalities. The mother had no significant disease and there was no history of exposure to embryopathic agents.

In our patient, the diagnosis was confirmed by biochemical and molecular genetic testing. Plasmalogen levels in fibroblasts of the patient were reduced compared to the reference values, and in addition no activity of the acyl-COA:dihydroxyacetone phosphate acyltransferase (DHAPAT) was measured, suggesting RCDP type 2. Mutation analysis of GNPAT (DHAPAT) gene identified a homozygous variant (c.1596_1597del) not previously reported in the literature.

Chondrodysplasia punctata (CDP), also known as multiple stippled epiphyses, is defined by the radiographic appearance of abnormal cartilaginous stippling either in the epiphyseal growth plate or in cartilaginous regions not usually expected to calcify [2]. When seen, one should first note whether rhizomelia is present in either the upper and/or lower extremities. CDP can be broadly divided into rhizomelic and non-rhizomelic forms.

RCDP are autosomal recessive conditions and are classified into five genetic subtypes. RCDP type 1 (OMIM 215100) and type 5, caused by mutations in the PEX7 and PEX5 gene, respectively, are peroxisome biogenesis disorders caused by a defect in the assembly of peroxisomes. RCDP type 2 (OMIM 22765), type 3 (OMIM 600121), and type 4 (OMIM 616107) are caused by a single peroxisomal enzyme deficiency [3].

RCDP type 1, 2, 3, and 5 are in most cases clinically and radiographically indistinguishable, while RCDP type 4 is atypical because patients were found to lack the characteristic symmetrical rhizomelic shortening of the long bones [3].

The main features of RCDP are rhizomelic shortening, mainly affecting the humerus and/or femurs, punctuate calcifications 
around joints and within cartilage (epiphyseal areas), contractures, microcephaly, dysmorphic face (prominent forehead, hypoplastic midface, anteverted nasal tip), and severe growth retardation. Cardiac defects and bilateral congenital cataract are common. Diagnosis can be suspected on clinical aspects and on radiological skeletal survey picture [1-3]. Affected children have a reduced lifespan, usually from respiratory complications, and marked neurodevelopmental delay [4].

A prompt recognition of the radiological pattern of RCDP may avoid unnecessary diagnostic tests. In fact, a finding of chondrodysplasia punctata (including stippling) has a somewhat limited differential diagnosis: maternal autoimmune diseases [5], warfarin embryopathy [6], chromosomal abnormalities, mucolipidosis type II, et al. [7]. Moth-eaten appearance of bones or limb abnormalities (asymmetry, more generalized limb reduction defects) also require to include in the differential diagnosis some rare defects of cholesterol biosynthesis $[8,9]$.

Acknowledgments The authors are grateful to the laboratory Genetic Metabolic Diseases of the Academisch Medisch Centrum (AMC) of the University of Amsterdam for DHAPAT enzymatic analysis on fibroblasts culture and molecular analysis of GNPAT gene.

\section{Compliance with ethical standards}

Conflict of interest statement All the authors have read and approved the manuscript. We confirm that each author has participated sufficiently in various sections to take public responsibility for its content. We declare that there are no conflicts of interest in relation to this paper.

\section{References}

1. Poll-The BT, Wanders RJA. Peroxisomal disorders. In: Blau N, Duran M, Gibson KM, Dionisi-Vici C, editors. Physician's guide to the diagnosis, treatment, and follow-up of inherited metabolic diseases. Berlin Heidelberg: Springer; 2014. p. 375-97.

2. Irving MD, Chitty LS, Mansour S, Hall CM. Chondrodysplasia punctata: a clinical diagnostic and radiological review. Clin Dysmorphol. 2008;17(4):229-41.

3. Klouwer FCC, Huffnagel IC, Ferdinandusse S, Waterham HR, Wanders $\mathrm{RJ}$, Engelen M, et al. Clinical and biochemical pitfalls in the diagnosis of peroxisomal disorders. Neuropediatrics. 2016;47(4):205-20.

4. White AL, Modaff P, Holland-Morris F, Pauli RM. Natural history of rhizomelic chondrodysplasia punctata. Am J Med Genet A. 2003; 118A(4):332-42.

5. Chitayat D, Keating S, Zand DJ, Costa T, Zackai EH, Silverman E, et al. Chondrodysplasia punctata associated with maternal autoimmune diseases: expanding the spectrum from systemic lupus erythematosus (SLE) to mixed connective tissue disease (MCTD) and scleroderma report of eight cases. Am J Med Genet A. 2008;146A(23):3038-53.

6. Wellesley D, Moore I, Heard M, Keeton B. Two cases of warfarin embryopathy: a re-emergence of this condition? Br J Obstet Gynaecol. 1998;105(7):805-6.

7. Nimmo G, Monsonego S, Descartes M, Franklin J, Steinberg S, Braverman N. Rhizomelic chondrodysplasia punctata type 2 resulting from paternal isodisomy of chromosome 1 . Am J Med Genet A. 2010;152A(7):1812-7.

8. Rossi M, Hall CM, Bouvier R, Collardeau-Frachon S, Le Breton F, Bucourt M, et al. Radiographic features of the skeleton in disorders of post-squalene cholesterol biosynthesis. Pediatr Radiol. 2015;458(7):965-76.

9. Royal SA. Taybi and Lachman's radiology of syndromes, metabolic disorders and skeletal dysplasias. 5th edn. Mosby Inc.; 2008. p. 901-910. 